\title{
Neural representation of consciously imperceptible speech sound differences
}

\author{
JEFFREY ALLEN, NINA KRAUS, and ANN BRADLOW \\ Northwestern University, Evanston, Illinois
}

\begin{abstract}
The concept of subliminal perception has been a subject of interest and controversy for decades. Of interest in the present investigation was whether a neurophysiologic index of stimulus change could be elicited to speech sound contrasts that were consciously indiscriminable. The stimuli were chosen on the basis of each individual subject's discrimination threshold. The speech stimuli (which varied along an $F 3$ onset frequency continuum from $/ \mathrm{da} /$ to $/ \mathrm{ga} /$ ) were synthesized so that the acoustical properties of the stimuli could be tightly controlled. Subthreshold and suprathreshold stimuli were chosen on the basis of behavioral ability demonstrated during psychophysical testing. A significant neural representation of stimulus change, reflected by the mismatch negativity response, was obtained in all but 1 subject in response to subthreshold stimuli. Grand average responses differed significantly from responses obtained in a control condition consisting of physiologic responses elicited by physically identical stimuli. Furthermore, responses to suprathreshold stimuli (close to threshold) did not differ significantly from subthreshold responses with respect to latency, amplitude, or area. These results suggest that neural representation of consciously imperceptible stimulus differences occurs and that this representation occurs at a preattentive level.
\end{abstract}

\section{Subliminal Perception: A Psychological Perspective}

Preconscious perception without conscious awareness is a subject of interest and controversy. In this investigation, preconscious perception describes physiological or neurological processes that occur without behavioral or conscious perception, whereas perception refers to an overt behavioral response to a stimulus. Although the phenomenon of visual subliminal perception is now generally accepted (Fried, MacDonald, \& Wilson, 1997; Skrandies \& Jedynak, 1999), disagreement continues to occur over the existence of subliminal perception in other sensory modalities - notably, the auditory. Evidence in favor of auditory subliminal perception is beginning to accumulate from the field of behavioral psychology. These studies have used physiological measures, such as heart rate and skin conductance levels, as indications of preconscious processing. Typical psychophysical experiments adjust the stimulus intensity-to-white-noise ratio so that the subject is unable to consciously identify any meaningful signal. The results of a study by Borgeat, Boissonneault, and Chaloult (1989) indicate that heart rate can fluctuate in response to preconscious activating suggestions, thereby supporting the notion that auditory stimulation at intensities below recognition threshold can influence physiological responses. A preconscious activating suggestion might be, for example, a word or phrase that

This research was supported by NIH-NIDCD-DC 01510 . The authors thank Trent Nicol for his significant contributions to this study. Correspondence concerning this article should be addressed to N. Kraus, Northwestern University, Auditory Neuroscience Laboratory, Frances Searle Building, 2299 North Campus Drive, Evanston, IL 60208 (e-mail: nkraus@nwu.edu). can elicit an emotional reaction but is below the intensity threshold for perceptual identification. Similar studies using equivalent methods of evaluation have supported these findings (Chakalis \& Lowe, 1992; Dixon, 1981; Kotze \& Møller, 1990). Semantic activation without conscious identification has been reviewed by Holender (1986).

Reports of auditory subliminal stimulation are significant because they support the notion that some form of auditory processing can occur during preconscious conditions. However, the results of the aforementioned studies are open to multiple interpretations with regard to preconscious perception of auditory stimuli without conscious awareness. First, the studies generally measure a response that is only indirectly related to auditory processing, such as heart rate or skin conductance. Second, the stimulus parameters that are used in studies such as these are generally not tightly controlled. Third, these studies do not typically measure an appropriately defined behavioral threshold - for example, chance-level performance. Finally, behavioral perception is not generally measured on an individual level, so the subthreshold stimulation for one subject may be completely perceptible to another. Consequently, evoked potentials measured during the presentation of precisely controlled stimuli, in individuals with well-defined perceptual thresholds, were used to provide further insight into preconscious processing.

\section{Subliminal Perception: A Biological Perspective}

Fried et al. (1997) have provided evidence for the existence of preconscious visual memory. Using the mean neural discharge rate of hippocampal neurons in awake 
humans as a measure of neural activity, Fried and colleagues demonstrated enhanced neural activity during the presentation of visual stimuli that the subject had seen before but denied (did not consciously remember) having seen. This study demonstrates a clear distinction between preconscious neural activity and behavioral perception.

Subliminal visual perception has been further reported from the perspective of perceptual learning in humans. Skrandies and Jedynak (1999) used subthreshold stereoscopic stimuli to induce perceptual learning of stereoscopic targets. A significant number of the subjects not only learned to see the targets when subthreshold stimuli were used, but also had accompanying changes in the patterns of neural activation in the visual cortex, as was demonstrated by evoked potential recordings, thereby forming a connection between subliminal perception and neurological activity.

In the auditory system, preconscious learning has been demonstrated by Tremblay and colleagues (Tremblay, Kraus, \& McGee, 1998). After training, significant changes in auditory-evoked responses that did not necessarily demonstrate a change in the behavioral perception of stimulus differences were shown in subjects, thus providing physiological evidence of learning before learning was evident behaviorally. These data suggest a separation between preconscious perceptual learning and functional behavior.

\section{Neurophysiologic Responses in Humans}

Scalp electrodes permit the study of aggregate neural responses from various nuclei. Important to this investigation is a cortical auditory-evoked potential called mismatch negativity, or MMN. MMN is a neurophysiologic response that reflects the brain's representation of a stimulus change in an ongoing stimulus sequence. It is elicited by the occurrence of a physically deviant stimulus in sequence with a series of homogenous, or standard, stimuli. The standard stimulus in the sequence occurs most of the time, whereas the deviant stimulus occurs only a small percentage of the time. As such, MMN provides an objective tool for assessing some of the fundamental aspects of speech perception. It has been elicited in response to frequency, intensity, duration, spatial, and phonemic changes (Aaltonen, Niemi, Nyrke, \& Tuhkanen, 1987; Kaukoranta, Sams, Hari, Hämäläinen, \& Näätänen, 1989; Kraus et al., 1996; Kraus, McGee, Sharma, Carrell, \& Nicol, 1992; Näätänen, 1990; Näätänen, Paavilainen, Alho, Reinikainen, \& Sams, 1989; Novak, Ritter, Vaughan, \& Wiznitzer, 1990). Research has suggested that the MMN originates in thalamocortical areas of the central auditory system (Alho, 1995; Csépe, Karmos, \& Molnár, 1987; Kaukoranta et al., 1989; Kraus, McGee, Carrell, et al., 1994; Kraus, McGee, Littman, Nicol, \& King, 1994; Näätänen \& Picton, 1987). The response is typically displayed as a negative polarity waveform centered at approximately $200-300 \mathrm{msec}$ after stimulus onset. The MMN is obtained by subtracting the response to the deviant stimuli when presented in an alone condition from the response to the identical deviant stimuli when they signal a change in a sequence of standard stimuli.

Several research investigations have studied the preattentive characteristics of MMN. The response has been shown to be elicited passively and does not require attention (Näätänen, 1991; Novak et al., 1990). These findings seem to be especially evident for changes in frequency. Frequency changes seem to be largely independent of attentional focus, suggesting that MMN has a preconscious component. MMN has furthermore been obtained in unconscious coma patients (Kane et al., 1996) and in anesthetized animal models (Csépe et al., 1987; Javitt, Steinschneider, Schroeder, Vaughan, \& Arezzo, 1994; Kraus, McGee, Carrell, et al., 1994; Kraus, McGee, Littman, et al., 1994), also supporting the preattentive characteristics of MMN.

\section{Physiologic Responses at Discrimination Threshold}

Group data elicited by pure tones suggest that MMN cannot be generated by tones below the frequency discrimination threshold (Sams, Paavilainen, Alho, \& Näätänen, 1985). These studies indicated that only a small MMN can be elicited at the discrimination threshold and that $\mathrm{MMN}$ amplitude increases as a function of the difference in frequency between standard stimuli and deviant stimuli (Tiitinen, May, Reinikainen, \& Näätänen, 1994).

Findings from this laboratory (Kraus et al., 1996; McGee, Kraus, \& Nicol, 1997) suggest that, at least in children, MMN of significant magnitude can be elicited by speech sound differences that are near discrimination threshold in individual subjects. Variants of the speech stimuli /da/ that were just perceptibly different have also been shown to elicit significant MMN responses (Kraus et al., 1993). However, the same speech stimuli were used to elicit responses in all the subjects. Thus, the relationship between an individual subject's behavioral perception and the underlying physiologic responses was not examined systematically. Nevertheless, MMN can be used as a tool to measure the electrophysiology of the cortex in response to speech stimuli that differ in minimal and well-specified acoustic features. Furthermore, these stimuli could be manipulated so that each subject is presented with stimulus pairs that are below a psychophysical discrimination threshold based on a behavioral task.

The purpose of this study was to determine whether neurophysiologic responses can occur to speech sounds that are acoustically different but not consciously perceived as different by the subject. A major goal was to provide information about the biology of sensory activity that is not consciously perceived. The results of this study also provide a more accurate understanding of the perceptual processes that the MMN response represents.

\section{METHOD}

\author{
Subjects \\ Twelve normal hearing adult subjects ( 6 males, 6 females, 22-28 \\ years of age) participated in the study. All the subjects had normal
}


hearing thresholds ( $<20 \mathrm{~dB}$ HL for $500-8000 \mathrm{~Hz}$ ) and no history of cognitive, learning, or attention problems. The experiments were carried out in an acoustically shielded room.

\section{Psychophysical Experiments}

Stimuli and Procedure for Behavioral Task 1. In order to precisely control the acoustic parameters of the stimuli, synthetic speech stimuli were used. Just noticeable differences (JNDs) were measured on a $/ \mathrm{da} /-\mathrm{ga} /$ continuum in which the third formant onset frequency was varied. Characteristics of the stimuli are shown schematically in spectrographic format in Figure 1. First, second, fourth, and fifth formant frequencies do not change between stimuli. The stimuli were synthesized using a Klatt (1980) digital speech synthesizer. Along the /da/-/ga/ continuum, $F 3$ onset frequency was varied between 2580 and $2190 \mathrm{~Hz}$ in 40 steps of $10-\mathrm{Hz}$ decrements. The values of the synthesis parameters between endpoints were interpolated linearly to generate the intermediate stimuli. Total stimulus duration was $100 \mathrm{msec}$.

Speech stimuli were presented binaurally at $75 \mathrm{~dB}$ SPL through headphones, using a parameter estimation by sequential tracking (PEST) paradigm (Taylor \& Creelman, 1967). The stimuli were presented at $75 \mathrm{~dB}$ SPL during all parts of the study. Thus, all the stimuli were detectable with respect to intensity. The procedure required the listener to be presented with two pairs of syllables, where the members of one pair were the same and the members of the other pair were different. The subject's task was to indicate whether the members of the first or those of the second pair of syllables were different from each other. Responses were elicited in a forced-choice format. The order of same and different pairs was randomized. During the presentation of a single syllable pair, the /da/ endpoint syllable $(F 3=2580 \mathrm{~Hz})$ was always presented first and served as the anchor stimulus. This stimulus was later used as the deviant stimulus in the electrophysiological portion of the study. The same pair was composed of the presentation of two identical anchor stimuli, whereas the different pair was composed of an anchor stimulus and an acoustically different stimulus. Variation along the $/ \mathrm{da} /-/ \mathrm{ga} / \mathrm{con}-$ tinuum occurred in accordance with the PEST algorithm, starting with pairs that were easy to discriminate and moving to more acoustically similar pairs on the basis of the subject's performance

\section{Stimulus continuum}
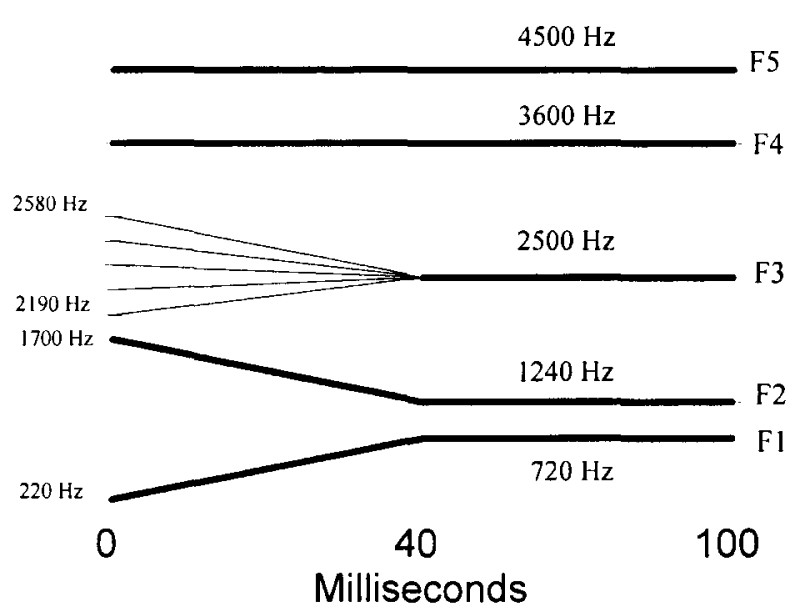

Figure 1. Spectrographic representation of stimuli. Characteristics common to all stimuli are represented by thick lines. $F 1, F 2$, $F 4$, and $F 5$ formant transitions do not change between stimuli. $F 3$ formant transitions for frequent stimuli varied between $2580 \mathrm{~Hz}$ (deviant/da/stimuli) and $2190 \mathrm{~Hz}$ (frequent/ga/stimuli). until an accuracy level of $69 \%$ correct was reached. If this level could not be obtained within 128 trials, the block was coded as a failure to converge. The subjects listened to at least three and as many as five experimental blocks, depending on the consistency of their results. The JND was computed as the mean JND of the two best blocks. All the subjects converged on at least three blocks (Carrell, Bradlow, Nicol, Koch, \& Kraus, 1999; Kraus, Koch, McGee, Nicol, \& Cunningham, 1999; Kraus et al., 1996).

Stimuli and Procedure for Behavioral Task 2. The premises of this investigation required an accurate assessment of which stimulus differences were indeed perceptible and of which were not perceptible. As such, an AX same-different discrimination paradigm was used, where $\mathrm{A}=$ anchor stimulus, $\mathrm{X}=$ variable stimulus, and the subject's task was to indicate whether A equaled or did not equal $X$. This task was different than the PEST task in that, instead of being presented with two syllable pairs, of which one consisted of two identical stimuli and the other consisted of two different stimuli, the subject was presented with only one syllable pair. The syllable pair was one of two types. A same trial consisted of either two identical anchor stimuli or two identical nonanchor stimuli. A different trial consisted of an anchor stimulus and an acoustically different stimulus. The subject's task was to indicate whether the two stimuli within the single syllable pair were the same or different from each other.

The stimulus set for this $\mathrm{AX}$ discrimination task was derived following and in conjunction with the PEST-determined JND scores so that only acoustic differences surrounding the JND were tested. That is, the stimulus continuum for this task was composed of 11 stimuli surrounding the JND ( 5 stimuli above the JND, 5 stimuli below the JND, and 1 stimulus at the JND). These 11 stimuli came from the same 40-step continuum used in the PEST task, in which each step represented a $10-\mathrm{Hz}$ change in $F 3$ onset frequency. In the event that 11 symmetrical stimuli could not be obtained owing to extremely low JND scores, a minimum of 11 total stimuli were still tested, with more above the JND than below.

The anchor stimulus in the pairs of different acoustic syllables was always the /da/ phoneme with an $F 3$ onset frequency of $2580 \mathrm{~Hz}$. The order of stimulus presentation within each trial was randomized. Pairing of the anchor (A) and different (X) stimuli occurred randomly, with the constraint that each of the 11 stimuli was paired with the anchor an equal number of times. These results were then averaged at each stimulus increment. Because each subject could theoretically have a unique JND, AX discrimination continua varied between subjects. Despite normal JNDs, some subjects had difficulty performing the AX same-different discrimination task. These subjects required the use of a stimulus continuum that included up to 16 stimuli.

Threshold determination. JND threshold was the point at which a discrimination accuracy of $69 \%$ was obtained. Using the PESTdetermined JND as an estimation, the AX discrimination task allowed refined assessment of discrimination accuracy surrounding the JND. A P(c)Max value that reflected discrimination accuracy could then be calculated.

The choice of the suprathreshold and subthreshold stimuli used in the neurophysiological experiments for each individual were based on both $P(\mathrm{c})$ Max values and JNDs. A suprathreshold response was defined as the point at which no less than $70 \%$ discrimination accuracy was obtained on the AX discrimination task and that exceeded the highest valid JND. A subthreshold response was defined as the point at which no greater than $59 \%$ discrimination accuracy was obtained on the AX discrimination task and that was less than the lowest valid JND score. These results allowed the neurophysiologic parameters for each subject to be set. For example, Subject 2 obtained JNDs of 59,47 , and $66 \mathrm{~Hz}(53-\mathrm{Hz}$ average), using the PEST protocol. The AX discrimination task was designed to test perception around these values. As such, stimulus pairs 1-2 through 1-12 were tested. A 1-2 stimulus refers to an anchor stimulus with 
an $F 3$ onset frequency of $2580 \mathrm{~Hz}$ and a variable stimulus $F 3$ onset frequency of $2570 \mathrm{~Hz}$, whereas a 1-12 stimulus pair refers to a $2580-\mathrm{Hz}$ anchor stimulus $F 3$ onset frequency and a $2470-\mathrm{Hz}$ variable stimulus $F 3$ onset frequency. Table 1 lists possible stimulus numbers with their corresponding $F 3$ onset frequency. $P(c) M a x$ values at each of the 11 increments between and including 1-2 through 1-12 were calculated. Subject 2 showed a $P(\mathrm{c})$ Max of $43.2 \%$ at stimulus increment $\mathrm{l}-3$ and a $P(\mathrm{c}) \mathrm{Max}$ of $80.6 \%$ at stimulus increment $1-11$. Because the $1-3$ contrast both was lower than the lowest JND and had a $P(\mathrm{c})$ Max value less than $59 \%$, it was chosen as the subthreshold stimulus pair. Likewise, the $1-11$ contrast was both higher than the highest JND obtained and greater than $70 \% P(\mathrm{c}) \mathrm{Max}$ and was therefore chosen as the suprathreshold stimulus pair.

\section{Neurophysiologic Experiments}

Stimuli and Procedure. Neurophysiological responses were obtained by using procedures that have been previously described (Kraus et al., 1999; Kraus, McGee, Carrell, \& Sharma, 1995; Kraus et al., 1996; Kraus et al., 1992). The stimuli were presented in an oddball paradigm, using the syllable/da/ with an $F 3$ onset frequency of $2580 \mathrm{~Hz}$ as the deviant stimulus in all conditions. Whereas the deviant /da/ stimulus remained constant, the standard /ga/ stimuli varied from subject to subject on the basis of their corresponding JND and discrimination scores. Electrophysiological responses were recorded from stimuli selected from the $/ \mathrm{da} /-\mathrm{ga} /$ continuum at suprathreshold and subthreshold intervals as described above. Presentation of the deviant $/ \mathrm{da} / \mathrm{stimuli}$ (probability of occurrence $=$ $10 \%$ ) occurred in a series of standard /ga/ stimuli (probability of occurrence $=90 \%$ ). The deviant $/ \mathrm{da} /$ stimulus was also presented in an alone condition. Because the standard/ga/ syllable varied during this experiment, the deviant/da/ syllable remained the same regardless of whether the contrast was subthreshold or suprathreshold. As such, acoustically identical deviant/da/stimuli were used to elicit MMN in both the suprathreshold and the subthreshold conditions. Only the context within which the deviant $/ \mathrm{da} /$ was presented varied.

Stimulus files from a Klatt synthesizer were downloaded to a PCbased stimulus delivery system that controlled time of delivery, stimulus intensity, and stimulus sequence. This system also triggered the PC-based evoked potential averaging system and indicated whether the trial contained a standard or a deviant stimulus. The stimuli were presented in a pseudorandom sequence, with at least three standard stimuli separating presentations of deviant stimuli. Twenty standard stimuli preceded the presentation of the first deviant stimuli. To control for level of arousal and to minimize the subject's attention to the stimuli, the subjects were instructed to watch videotapes. The videotape audio levels were kept below $40 \mathrm{~dB}$ SPL. Speech stimuli were presented to the right ear at $75 \mathrm{~dB}$ SPL through headphones.

Evoked responses elicited by standard stimuli and deviant stimuli were averaged separately. A minimum of 4,000 sweeps (deviant stimuli + standard stimuli) were collected for each subject. Those subjects who were exceptionally noisy were asked to repeat the neuro-

Table 1

Stimulus Number and $F 3$ Onset Frequency (in Hertz)

\begin{tabular}{cccc}
$\begin{array}{c}\text { Stimulus } \\
\text { Number }\end{array}$ & $\begin{array}{c}F 3 \text { Onset } \\
\text { Frequency }\end{array}$ & $\begin{array}{c}\text { Stimulus } \\
\text { Number }\end{array}$ & $\begin{array}{c}F 3 \text { Onset } \\
\text { Frequency }\end{array}$ \\
\hline 1 & 2580 & 11 & 2480 \\
2 & 2570 & 12 & 2470 \\
3 & 2560 & 13 & 2460 \\
4 & 2550 & 14 & 2450 \\
5 & 2540 & 15 & 2440 \\
6 & 2530 & 16 & 2430 \\
7 & 2520 & 17 & 2420 \\
8 & 2510 & 18 & 2410 \\
9 & 2500 & 19 & 2400 \\
10 & 2490 & 20 & 2390 \\
\hline
\end{tabular}

physiologic portion of the study, resulting in the collection of up to 8,000 sweeps (deviant stimuli + standard stimuli). In addition, there was a deviant-alone condition in which responses to approximately 1,500 stimulus presentations of the deviant stimuli presented alone were collected.

Evoked potentials were recorded from 12 scalp locations. For all the stimulus contrasts, the forehead electrode served as the ground. Eye blink movements were recorded from the left eye by two separate electrodes. When eye blinks occurred, the evoked potential responses were deleted from the averaged response. Active electrodes were placed at locations $\mathrm{Fz}, \mathrm{Cz}, \mathrm{Pz}, \mathrm{F} 3, \mathrm{~F} 4, \mathrm{~A} 1$, and A2. Temporal lobe activity was recorded by electrodes TL and TR. These electrodes were located halfway between T4 and T6 (TR) and between T3 and T5 (TL). Electrode placements are within the regulations established by the International 10/20 recording system (Jasper, 1958). In addition, an electrode was placed on the nose to serve as a reference.

\section{Data Analysis}

Individual subject analysis. Because $M M N$ is elicited by the deviant stimulus only when it signals an acoustic change, difference waves were computed for each subject by subtracting the response to the deviant stimulus presented alone from the response to the same deviant stimulus presented within the oddball paradigm (Kraus et al., 1995). Likewise, a control difference wave was obtained in a condition in which no MMN should exist: the difference between responses to two (identical) standard stimuli presented immediately before the deviant stimuli.

MMN responses were identified visually in the difference waves as a relative negativity following the $\mathrm{N} 100$, occurring between 100 and $500 \mathrm{msec}$ after stimulus presentation. Onset latency was defined as the first negative deviation from baseline, and the offset latency was the return to baseline. Onset, peak, and offset latencies of MMN were measured. MMN duration was computed by subtracting the onset latency from the offset latency. For a few subjects, there were two negative deflections from baseline. For these subjects, the total duration was computed as the sum of the duration of each negative deflection. Amplitude of onset to peak latency was measured. MMN area was computed by integrating the overall response area between the onset and the offset points.

An analysis of MMN significance in individual subjects was performed by using the $Z$ test as a measure of significance. The suprathreshold and subthreshold conditions were compared with the control condition for significance. $Z$ scores were calculated by comparing an individual value with the mean and standard deviation of a comparison control condition. In this case, the MMN area of each individual subject was compared with the mean and standard deviation of the area of the control difference wave. The control difference wave was obtained by subtracting the response to the standard stimuli from the response to the standard stimuli presented immediately before the rare stimuli. Because no stimulus difference occurs in this case by definition, no MMN is generated. That is, any "response" occurring in the region of interest $(100-500 \mathrm{msec})$ must be spurious noise. By measuring this control condition noise, it was possible to define a "response" that a true MMN must exceed. This was accomplished by computing means and standard deviations of the various parameters (area, duration, etc.) in the control condition to establish a confidence level beyond which a response could be considered significant. Control means and standard deviations were calculated only on the basis of waveforms that showed some measurable response. That is, responses showing no measurable waveforms (zeros) were excluded. This is a conservative method for determining whether a real response occurred, because inclusion of waveforms with no response would have made the criteria for a true response more lenient.

Group data analysis. The data were also analyzed across subjects. For both the suprathreshold and the subthreshold stimulus conditions, each subject's data were used to compute means and 
standard deviations of group MMN onset, offset, duration, amplitude, and area. For each of these parameters, $t$ tests were performed in order to compare the suprathreshold, subthreshold, and control conditions. Amplitude and latency of Pl/N1 group responses were also analyzed. $\mathrm{Pl} / \mathrm{N} 1$ responses during suprathreshold stimulation and subthreshold stimulation were compared by using the $t$ test.

In addition, grand average responses were computed across subjects for the subthreshold, suprathreshold, and control conditions. A point-to-point $t$ test was used to denote the portion of the waveform that was significantly different from zero (Kraus et al., 1995). This type of analysis has the advantage of avoiding subjective identification of peaks.

\section{RESULTS}

\section{Behavioral Analysis}

JND results and $P(\mathrm{c})$ Max values for individual subjects are presented in Table 2. The table indicates the highest, lowest, and average JND responses that were obtained by each subject. JND scores are reported in hertz and refer to the $F 3$ onset frequency difference between the anchor /da/ stimulus and the variable /ga/ stimulus. $P(\mathrm{c})$ Max values of the subthreshold and suprathreshold stimuli that were used in the electrophysiological portion of the study are also reported. The average $P(\mathrm{c}) \mathrm{Max}$ value for the subthreshold condition was 50.8, indicating chance discrimination (chance discrimination $=50 \%$ ). The average $P(\mathrm{c})$ Max value for the suprathreshold condition was 74.5 , indicating an adequate ability to distinguish the stimulus pairs. Stimulus pairs used for each subject are also reported in Table 2. Table 1 indicates the stimulus $F 3$ onset frequency that is represented by each stimulus number. All of the stimuli selected for each subject follow the criteria established in the Method section of the study.

\section{Electrophysiological Group Analysis}

Mismatch negativity group analysis. Figure 2 shows the grand average responses to the standard stimulus and the deviant stimulus for the suprathreshold condition (top), the subthreshold condition (middle), and the control condition (bottom). In each panel, the top thin trace is the response to the deviant stimulus when presented alone, the thick trace is the response to the deviant stimulus when presented in the oddball paradigm, and the bottom trace represents the grand average MMN difference wave. The difference wave is obtained by subtracting the response to the same stimulus presented in both the alone and the oddball conditions. Boxed regions beneath the difference wave denote the portion of the wave that is significantly different from zero $[t(11)=1.81, p<$ $.05]$. A significant $M M N$ is clearly shown in both the subthreshold and the suprathreshold conditions. A similar analysis was performed on the control (standard stimulus standard stimulus) condition. No regions of significance were found.

Average responses and statistical measures of latency, amplitude, and area are shown in Table 3. Table 4 provides parametric $t$ tests of area, latency, and amplitude, comparing the suprathreshold and the subthreshold conditions with each other and with the control (same stimulus). All calculations comparing the subthreshold condition with the control and the suprathreshold condition to control were significant $(p<.05)$. Although the peak area and peak amplitude were greater and the average duration was shorter for the suprathreshold condition than for the subthreshold condition, no significant differences between these two conditions were found.

$P 1 / N 1$ group analysis. $P 1$ and $N 1$ responses to the frequent/ga/stimuli were recorded during both stimulus conditions. There were no significant differences between $\mathrm{P} 1 / \mathrm{N} 1$ parameters elicited by the different $/ \mathrm{ga} / \mathrm{stim}$ uli $[t(11)=1.81, p<.05]$ or between the responses to $/ \mathrm{da} /$ in the deviant conditions (Figure 2). Parameters measured were the latencies of the P1 onset and the $\mathrm{N} 1$ onset and P1 to N1 latency. Amplitude measures from baseline to Pl and from Pl to N1 were also analyzed.

Table 2

Just Noticeable Difference (JND) Values, $P(c) M a x$ Values, and Stimulus Pairs Obtained During Behavioral Testing in All Subjects

\begin{tabular}{|c|c|c|c|c|c|c|c|}
\hline \multirow[b]{2}{*}{ Subject } & \multicolumn{3}{|c|}{$\mathrm{JND}(\mathrm{Hz})$} & \multicolumn{2}{|c|}{$P(\mathrm{c})$ Max } & \multicolumn{2}{|c|}{ Stimulus Pairs } \\
\hline & Average & Lowest & Highest & Sub & Supra & Sub & Supra \\
\hline 1 & 50 & 47 & 72 & 57.5 & 70.1 & $1-4$ & $1-10$ \\
\hline 2 & 53 & 47 & 66 & 43.2 & 80.6 & $1-3$ & $1-11$ \\
\hline 3 & 38 & 28 & 47 & 49.2 & 77.0 & $1-2$ & $1-9$ \\
\hline 4 & 95 & 72 & 128 & 55.5 & 78.5 & $1-6$ & $1-14$ \\
\hline 5 & 65 & 59 & 72 & 57.9 & 80.0 & $1-3$ & $1-9$ \\
\hline 6 & 72 & 47 & 128 & 49.2 & 71.2 & $1-4$ & $1-14$ \\
\hline 7 & 53 & 47 & 72 & 34.9 & 73.1 & $1-4$ & $1-16$ \\
\hline 8 & 57 & 47 & 72 & 56.6 & 70.2 & $1-3$ & $1-11$ \\
\hline 9 & 44 & 34 & 53 & 45.8 & 74.0 & $1-2$ & $1-11$ \\
\hline 10 & 75 & 53 & 122 & 49.0 & 71.0 & $1-3$ & $\mathbf{1}-12$ \\
\hline 11 & 102 & 72 & 138 & 57.1 & 71.1 & $1-5$ & $1-17$ \\
\hline 12 & 70 & 59 & 122 & 53.4 & 76.6 & $1-5$ & $1-13$ \\
\hline Average & 65 & 51 & 91 & 50.8 & 74.5 & $1-3.7$ & $1-12.3$ \\
\hline
\end{tabular}

Note-JND values listed are the average, lowest obtainable JND and the highest obtainable JND. P(c)Max values shown are for the respective subthreshold (Sub) and suprathreshold (Supra) stimuli. Stimulus pairs used in the electrophysiological portion of the study, as well as group average values for all measures, are also listed. 

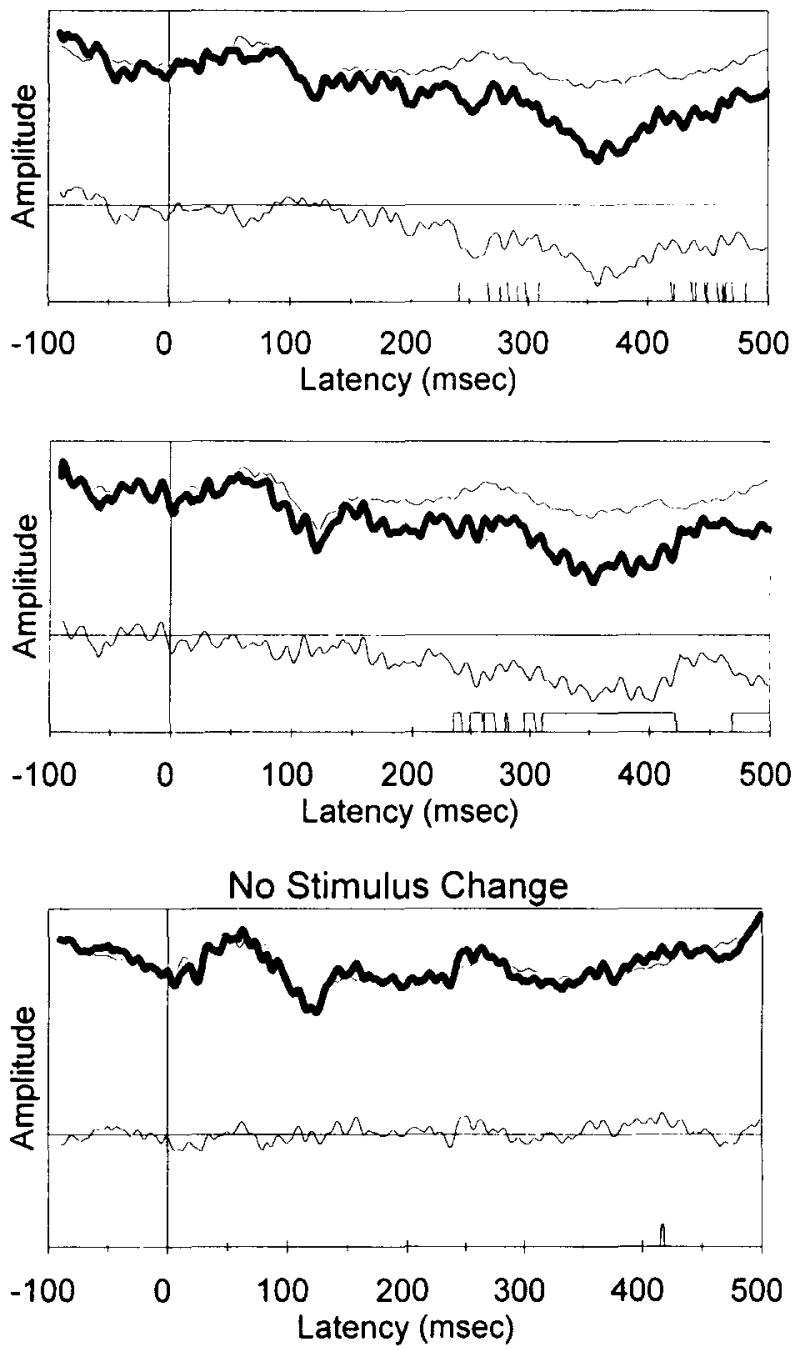

Figure 2. Grand average responses to suprathreshold (top), subthreshold (middle), and control (bottom) conditions. In the suprathreshold and subthreshold graphs, the top thin trace represents the response to the /da/deviant stimulus presented alone. The middle thick trace represents the response to the/da/deviant stimulus when presented in the oddball paradigm. The bottom difference wave was obtained by subtracting the response to /da/ deviant alone from $/ \mathrm{da} /$ deviant when it was the deviant stimulus in the oddball paradigm. Significant mismatch negativity differences are indicated by the boxes under the difference waves $[t(11)=1.81, p<.05]$.

\section{Electrophysiological Individual Subject Analysis}

Suprathreshold and subthreshold conditions versus control. An analysis of MMN significance in individual subjects is found in Table 5. A $Z$ score was used as a measure of individual MMN area significance. Significant MMN $[Z(11)>1.96, p<.05]$ was detected in all the subjects in the suprathreshold condition and all but one subject (Subject 7) in the subthreshold condition. The MMN response is typically evident in the response to the deviant stimulus approximately $200-300 \mathrm{msec}$ following stimulus presentation. All of the figures shown and calculations performed with respect to the electrophysiological analysis are taken from data collected at the Fz electrode. This was representative of activity that occurred at other electrode locations.

Subthreshold condition versus suprathreshold condition. Individual subject MMN area, duration, and amplitude are shown in Figure 3. For each parameter, suprathreshold and subthreshold data are shown next to each other. Figure 4 depicts individual onset and offset MMN responses for both conditions. Graphic representation of all the parameters does not show any significant differences between suprathreshold and subthreshold conditions.

\section{DISCUSSION}

\section{Preconscious Auditory Perception}

This study clearly demonstrated that neural responses can be obtained to stimuli that are not consciously discriminated. This suggests that fine-grained auditory processing can occur in the absence of conscious perception of stimulus differences. That is, the brain may produce a code of subtle sound changes that cannot be behaviorally discriminated. Put another way, this code cannot be read at higher levels of sound processing in the brain and therefore remains subliminal. Thus, behavioral perception does not necessarily match the preconscious neural representation of the same physical stimulus event. Overall, the findings imply that sensory processes may be more precise than we think (i.e., better than one is consciously aware of).

The existence of nervous system representation of subliminal sensory events is relevant to our understanding of perception in general. For example, it is likely that the integration of perceptual information as a unitary auditory event or object depends on subliminal perceptual processes. The results here support previous findings allud-

Table 3

Average Mismatch Negativity Data for Latency, Amplitude, and Area

\begin{tabular}{lrrrrr}
\hline & \multicolumn{2}{c}{ Suprathreshold } & & \multicolumn{2}{c}{ Subthreshold } \\
\cline { 2 - 3 } \multicolumn{1}{c}{ Parameter } & Average & $S D$ & & Average & $S D$ \\
\hline Latency $(\mathrm{msec})$ & & & & \\
Onset & 201.06 & 51.36 & & 194.85 & 52.06 \\
Offset & 450.29 & 41.13 & & 468.31 & 26.89 \\
Duration & 237.77 & 49.32 & & 255.43 & 45.44 \\
Amplitude $(\mu \mathrm{V})$ & 2.25 & 0.78 & & 2.04 & 0.64 \\
Area $(\mu \mathrm{V} \cdot \mathrm{msec})$ & 307.45 & 80.71 & & 303.04 & 89.04 \\
\hline
\end{tabular}

Table 4

Parametric Paired $t$ Tests for Significance of Area, Duration, and Amplitude Comparing Supratheshold and Subthreshold Conditions to Each Other and to the Control (Same Stimulus) Condition

\begin{tabular}{lccc}
\multicolumn{1}{c}{ Condition } & Area & Duration & Amplitude \\
\hline Suprathreshold versus control & $\mathbf{1 . 7 E - 0 5}$ & $\mathbf{2 . 2 E - 0 5}$ & $\mathbf{2 . 7 E - 0 4}$ \\
Subthreshold versus control & $\mathbf{1 . 4 E - 0 5}$ & $\mathbf{2 . 8 E - 0 6}$ & $\mathbf{2 . 4 E - 0 4}$ \\
Suprathreshold versus subthreshold & .527 & .747 & .938 \\
\hline
\end{tabular}

Note-Boldface values are significant $(p<.05)$. 
Table 5

Analysis of Mismatch Negativity Significance in Individual Subjects

\begin{tabular}{ccc} 
& \multicolumn{2}{c}{$Z$ Test Comparison Condition } \\
\cline { 2 - 3 } Subject & $\begin{array}{c}\text { Suprathreshold- } \\
\text { Control (Same Stimulus) }\end{array}$ & $\begin{array}{c}\text { Subthreshold- } \\
\text { Control (Same } \text { Stimulus) }\end{array}$ \\
\hline 1 & 2.7 & $\mathbf{4 . 6}$ \\
2 & 6.5 & 7.1 \\
3 & 6.1 & 4.3 \\
4 & 4.3 & 5.7 \\
5 & 4.4 & 3.1 \\
6 & 4.9 & 7.7 \\
7 & 3.3 & 1.7 \\
8 & 7.6 & 3.7 \\
9 & 5.8 & 5.5 \\
10 & 4.9 & 4.6 \\
11 & 9.2 & 6.3 \\
12 & 3.1 & $\mathbf{2 . 9}$
\end{tabular}

Note-Area data are used as the measure of significance in this analysis. $Z$ scores greater than 1.96 (boldface) are significant.

ing to the existence of a neural representation of stimulus events that are not consciously perceived or remembered (Fried et al., 1997; Holender, 1986; Tremblay et al., 1998). The data also suggest that it may be possible to access this information with training that improves perception (Tremblay et al., 1998).

A potential limitation of this study is that our determination of a subthreshold contrast is limited by the sensitivity of the task used. It is possible that other psychophysical tasks might have revealed sensitivity to stimulus contrasts that our 4IAX and AX tasks masked. (For performance differences across various psychophysical testing paradigms, see Macmillan \& Creelman, 1991.) It may be that, under certain test conditions, our subthreshold stimulus pairs were in fact behaviorally discriminable. Nevertheless, it was critical for our study that the behavioral and physiologic test paradigms were well matched, and this requirement was met by the behavioral tasks we used.

Furthermore, there are several reasons that lead us to say with confidence that the subjects with relatively good psychophysical performance (who might have perceived the subthreshold stimuli) were not responsible for the overall effect. There is no rank-order correlation between the subthreshold $z$ scores from Table 5 and the subthreshold $P(\mathrm{c})$ Max from Table $2(\rho-.073, p=0.81)$. All but one subject showed significant physiologic responses in the subthreshold condition (Table 5), and an analysis of the subthreshold grand average neurophysiologic data minus the subjects with $\mathrm{A}-\mathrm{X}$ scores in the $56 \%-57 \%$ range continued to produce a significant MMN. Thus, it is not the case that the subjects with the best perception of the subthreshold stimuli had larger MMNs that exaggerated average subthreshold MMN across all subjects.

This investigation is consistent with the notion that the neural generators responsible for the MMN are not necessarily linked to conscious perception (Näätänen, 1991). It is highly unlikely that listeners were attending to the auditory stimuli during MMN testing. The stimulus contrasts under investigation were barely detectable (or not detected at all) in the quiet, controlled environment of the psychophysical experiment in which the subject's task was to carefully attend to the stimuli. During MMN recording, not only was the subject's attention distracted by the video (which was inherently more interesting than the speech syllables), but subjects listened to the soundtrack of the video, which further mitigated against the subject's attending to stimulus differences during physiologic recording. Furthermore, test-retest of MMN shows little change (Escera \& Grau, 1996; Frodl-Bauch, Kathmann, Moller, \& Hegerl, 1997; Pekkonen, Rinne, \& Näätänen, 1995; Tervaniemi et al., 1999), making it un-
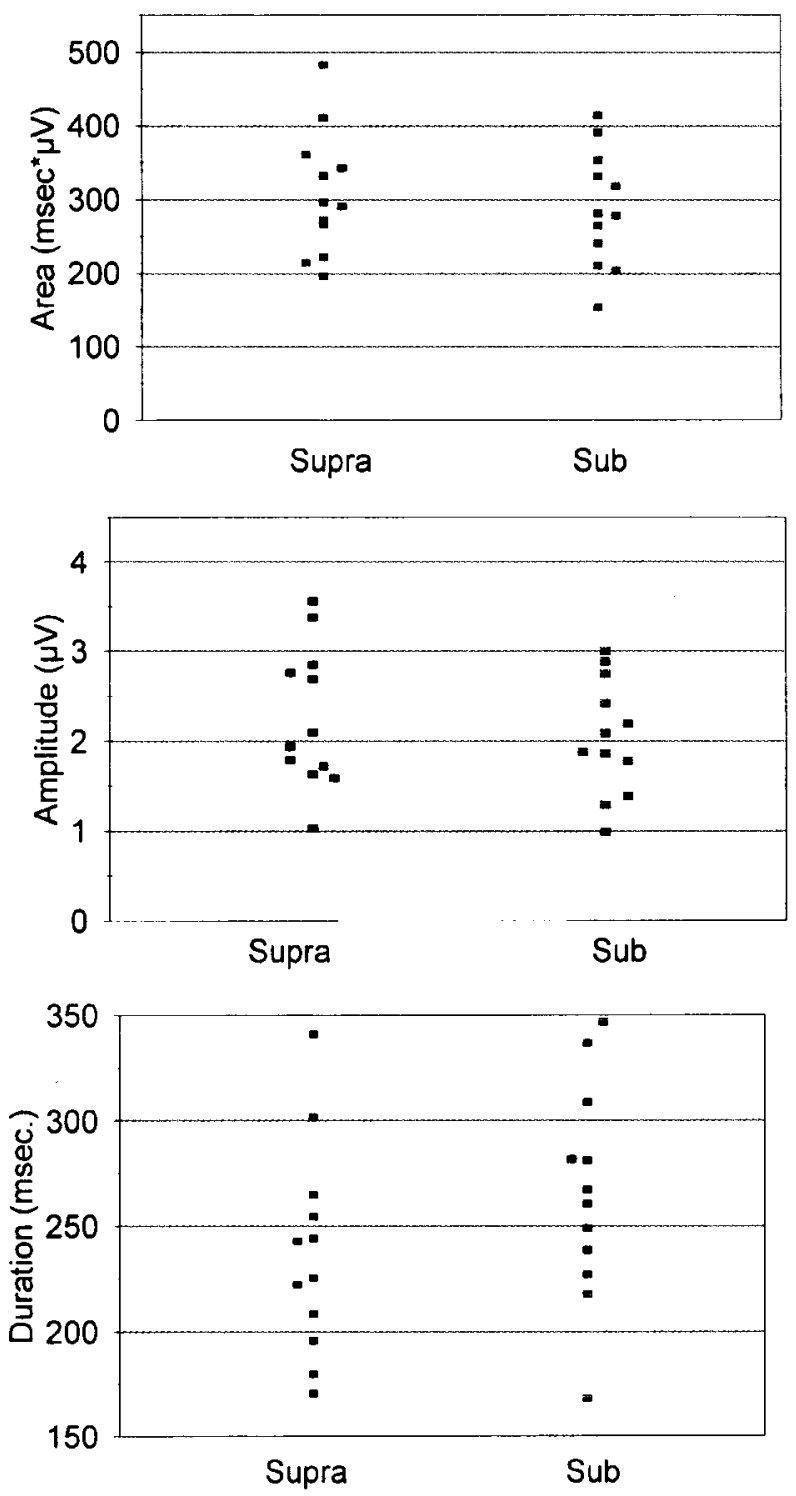

Figure 3. The area (top), duration (bottom), and amplitude (middle) of mismatch negativity (MMN) response are shown for each subject during both stimulus conditions. 


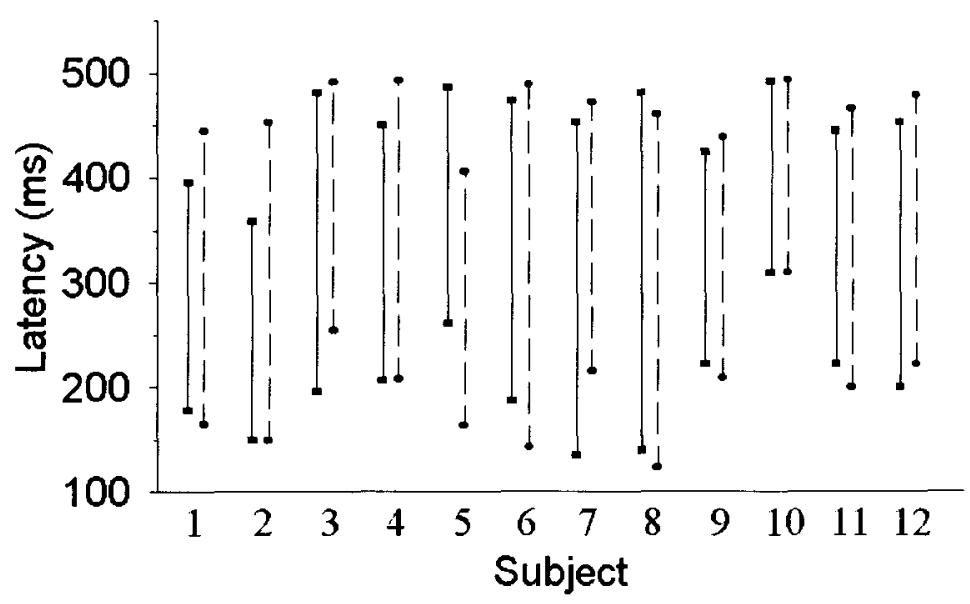

Figure 4. The onset and offset of mismatch negativity is compared in the suprathreshold (solid line, squares) and subthreshold (dotted line, circles) conditions for each subject.

likely that passive exposure to the stimuli caused the brain to respond to stimuli to which it was previously (behaviorally) unresponsive. Consequently, MMN provides a way to probe preconscious auditory sensory processes in humans. This contrasts with other neurophysiologic reflections of stimulus differences, such as $\mathrm{P} 300$, which occur only in representations of stimulus differences that people can hear (Hoffman, Houck, MacMillan, Simons, \& Oatman, 1985; Lang, Lang, Heise, Deecke, \& Kornhuber, 1984).

Some studies have suggested that MMN is a necessary but not a sufficient component for conscious perception of a stimulus change (Näätänen, Gaillard, \& Mantysalo, 1978). However, it has been documented that some individuals do not have MMN despite behavioral perception of the stimulus change used to elicit the representation, suggesting that conscious perception of acoustic stimulus differences may not require whatever processes are responsible for MMN generation (Kraus et al., 1999). It is important to realize that $\mathrm{MMN}$ and behavioral responses to the same signals represent different aspects of signal processing, the former being preattentive and neurobiological, whereas the latter involves the conscious integration of perceptual information. Nevertheless, biological and perceptual processes that govern how we hear speech may be better understood by understanding how preattentive neural processes and conscious perception are related to one another.

\section{Suprathreshold and Subthreshold Mismatch Negativity Similarity}

The MMN is thought to be an extremely sensitive neurophysiologic response to physical stimulus differences in complex speech signals (Kraus et al., 1993; Sams et al., 1985; Sharma, Kraus, McGee, Carrell, \& Nicol, 1993). The results of the present study support this statement. As was expected, the suprathreshold condition produced significant MMN with respect to duration, amplitude, and area. It was not necessarily expected, however, that a subthreshold response of similar duration, amplitude, and area as the suprathreshold response would be elicited. Several explanations might explain the similarity between the MMNs obtained in the two conditions. First, $\mathrm{P} 3 \mathrm{a}$ is a response that, like MMN, is elicited by stimulus change at approximately $300 \mathrm{msec}$ poststimulus (Escera, Alho, Winkler, \& Näätänen, 1998; Polich, 1988; Squires, Squires, \& Hillyard, 1975). P3a has been shown to be a graded response that increases in magnitude as stimulus differences increase (Comerchero \& Polich, 1998). Because $\mathrm{P} 3 \mathrm{a}$ is a positivity and $\mathrm{MMN}$ is a negativity, these responses may have a canceling effect on each other. On account of its greater stimulus difference, the suprathreshold condition would be most affected by P3a, thereby producing reduced $\mathrm{MMN}$, resulting in the apparent similarity in suprathreshold and subthreshold responses. Some studies have shown that increasing the magnitude of stimulus deviation results in a MMN that is shorter in duration and larger in area (Näätänen et al., 1989; Novak et al., 1990; Sams et al., 1985). The results of the present study show a similar trend in these parameters, although they fail to show significant differences.

Alternatively, the similarity between suprathreshold MMN and subthreshold MMN may indicate that nearthreshold stimuli produce similar responses. Even for subjects who had a high perceptual threshold, the subthreshold and suprathreshold stimuli differed by no more than $120 \mathrm{~Hz}$. For some subjects, the stimulus differences were as small as $60 \mathrm{~Hz}$. It is conceivable that because the suprathreshold and the subthreshold stimulus pairs used were not very different from each other, the resulting MMNs were not very different from each other. Furthermore, the response similarity might not have been a function of perceptual ability but, rather, a function of the physical similarities of the stimulus conditions. Sharma 
et al. (1993) reported similar MMNs to phonemes that were across and within categorical boundaries, even though the two phonemes differed in identical magnitudes of $F 3$ onset frequency. That is, the behavioral experience of the subject played an insignificant role in the magnitude and duration of MMN. The findings of the present study support those obtained by Sharma et al. in terms of behavioral experience and MMN magnitude.

For future consideration, one might wish to design a study using a paradigm similar to the present study, with the addition of stimulus pairs that differ by the same amount as the suprathreshold and subthreshold conditions but that are both perceptually different from the anchor stimuli. So, for example, when an anchor stimulus of $2580 \mathrm{~Hz}$ is used and subthreshold and suprathreshold stimuli are determined to be 2530 and $2430 \mathrm{~Hz}$, respectively, the difference between subthreshold and suprathreshold stimuli is $100 \mathrm{~Hz}$. Recording the same response at $2330 \mathrm{~Hz}$ might help determine how the measured responses are related to the physical properties of the stimulus and whether the conscious threshold of perception was influencing the responses. If it can be shown that the similarity in the stimulus pairs used accounts for the MMN similarity, one might speculate as to what significance the perceptual threshold has in terms of the MMN. That is to say, MMN production might be independent of behavioral experience, including conscious perception.

\section{P1/N1 Effects}

It is also worth mentioning that $\mathrm{P} 1 / \mathrm{N} 1$ parameters elicited by the different /ga/ stimuli were not significantly different. This result is important because it supports the notion that the neural generators responding to the intrinsic acoustic properties of the stimuli (separate from stimulus change) were similar in both conditions, thereby suggesting that MMN production was not biased by unequal $\mathrm{N} 1$ responses. Moreover, the fact that MMN was obtained to stimulus change, whereas $\mathrm{P} 1 / \mathrm{Nl}$ was not, is consistent with the notion that intrinsic acoustic characteristics and acoustic change are represented by distinct neural pathways and that $\mathrm{N} 1$ and MMN reflect different processes (Escera et al., 1998; Näätänen \& Picton, 1987; Sams, Kaukoranta, Hämäläinen, \& Näätänen, 1991).

\section{Clinical Relevance}

Because it is possible to obtain a neurophysiologic reflection of preconscious processing of stimulus differences, one can record sensory perception below the level of that one can consciously perceive. The results provided here indicate that sensory perception may be better than we are consciously aware. The ability to access this nervous system event may provide a way to monitor brain changes throughout the course of behavioral training. This could be useful when training nonnative speakers to understand fine acoustic differences in a foreign language (Bradlow, Pisoni, Akahane-Yamada, \& Tohkura, 1997; Flege, 1995; Jamieson \& Morosan, 1986; Logan \& Pruitt, 1995; Strange \& Dittmann, 1984; Tremblay, Kraus,
Carrell, \& McGee, 1997) or when assisting individuals with learning disabilities to discriminate fine acoustic differences (Godfrey, Syrdal-Lasky, Millay, \& Knox, 1981; Kraus et al., 1996; Tallal \& Stark, 1981; Werker \& Tees, 1987). Moreover, because MMN appears to reflect a preconscious neural representation of stimuli, it might be expected that in an individual who did not have this neurophysiologic response to stimulus change, the neural mechanisms leading up to preconscious processing would be faulty, and not necessarily those mechanisms that take a preconscious signal and allow it to become a conscious event. As such, a clinician treating a disorder manifesting from a faulty MMN-generating system might be better able to pinpoint the neural processes responsible for the disorder.

When using any measure of evaluation, it is important to have values that represent the normal expectations of the measuring device. In this respect, this study is significant because it has helped determine what sort of response one might expect when the psychophysical limits of any single individual are reached.

\section{Future Research}

When two acoustically identical stimuli are presented to a subject, MMN does not occur. Some sort of perceptual limit must therefore exist. That is, there must be some point along the continuum between the subthreshold stimulus differences used here and the point at which they become identical at which the neural representation of physically different stimuli ceases to exist. It might be interesting to investigate how far below threshold one must go before a physical difference fails to be represented by the central nervous system. Likewise, the point above threshold at which the neurophysiologic response becomes larger with increasing stimulus differences would also be interesting to determine. These studies might help clarify the relationship between the behavioral (conscious) discrimination threshold and the threshold of stimulus differences by which neurophysiologic representation of stimulus change is dictated.

\section{CONCLUSION}

This study indicates that (1) neural representation of consciously imperceptible auditory stimulus differences can occur, (2) stimulus differences that are below discrimination threshold produce $\mathrm{MMN}$ responses that are similar to responses elicited by stimuli that are just above discrimination threshold, and (3) it is possible to access neurobiologic processes that are largely independent of conscious perception in order to probe preconscious auditory perception in humans.

\section{REFERENCES}

Aaltonen, O., Niemi, P., Nyrke, T., \& Tuhranen, M. (1987). Eventrelated brain potentials and the perception of a phonetic continuum. Biological Psychology, 24, 197-207.

ALHо, K. (1995). Cerebral generators of mismatch negativity (MMN) 
and its magnetic counterpart (MMNm) elicited by sound changes. Ear \& Hearing, 16, 38-51.

Borgeat, F., Boissonneault, J., \& Chaloult, L. (1989). Psychophysical responses to subliminal auditory suggestions for activation. Perceptual \& Motor Skills, 69, 947-953.

Bradlow, A. R., Pisoni, D. B., Akahane-Yamada, R., \& Tohkura, Y. (1997). Training Japanese listeners to identify English /r/ and /1/: IV. Some effects of perceptual learning on speech production. Journal of the Acoustical Society of America, 101, 2299-2310.

Carrell, T. D., Bradlow, A. R., Nicol, T. G., Koch, D. B., \& KraUS, N. (1999). Interactive software for evaluating auditory discrimination. Ear \& Hearing, 20, 175-176.

Chakalis, E., \& Lowe, G. (1992). Positive effects of subliminal stimulation on memory. Perceptual \& Motor Skills, 74, 956-958.

Comerchero, M. D., \& Polich, J. (1998). P3a, perceptual distinctiveness, and stimulus modality. Brain Research: Cognitive Brain Research, 7, 41-48.

CSÉPE, V., Karmos, G., \& Molnár, M. (1987). Evoked potential correlates of stimulus deviance during wakefulness and sleep in the catanimal model of mismatch negativity. Electroencephalography \& Clinical Neurophysiology, 66, 571-578.

Dixon, N. F. (1981). Preconscious processing. New York: Wiley.

Escera, C., Alho, K., Winkler, I., \& NäÄTÄnen, R. (1998). Neural mechanisms of involuntary attention to acoustic novelty and change. Journal of Cognitive Neuroscience, 10, 590-604.

EsCERA, C., \& GraU, C. (1996). Short-term replicability of the mismatch negativity. Electroencephalography \& Clinical Neurophysiology, 100, 549-554.

FLEGE, J. E. (1995). Two procedures for training a novel second language phonetic contrast. Applied Psycholinguistics, 16, 425-442.

Fried, I., MacDonald, K. A., \& Wilson, C. L. (1997). Single neuron activity in human hippocampus and amygdala during recognition of faces and objects. Neuron, 18, 753-765.

Frodl-Bauch, T., KathmanN, N., Moller, H. J., \& Hegerl, U. (1997). Dipole localization and test-retest reliability of frequency and duration mismatch negativity generator processes. Brain Topography, 10, 3-8.

Godfrey, J. J., Syrdal-Lasky, A. K., Millay, K. K., \& Knox, C. M. (1981). Performance of dyslexic children on speech perception tests. Journal of Experimental Child Psychology, 32, 401-424.

Hoffman, J. E., Houck, M. R., MacMillan, F. W., III, Simons, R. F., \& OATMAN, L. (1985). Event-related potentials elicited by automatic targets: A dual-task analysis. Journal of Experimental Psychology: Human Perception \& Performance, 11, 50-61.

HOLENDER, D. (1986). Semantic activation without conscious identification in dichotic listening, parafoveal vision, and visual masking: A survey and appraisal. Behavioral \& Brain Sciences, 9, 1-66.

Jamieson, D. G., \& Morosan, D. E. (1986). Training non-native speech contrasts in aduits: Acquisition of the English $/ \delta /-/ \theta /$ contrast by francophones. Perception \& Psychophysics, 40, 205-215.

JASPER, H. H. (1958). The ten-twenty electrode system of the international federation. Electroencephalography \& Clinical Neurophysiology, 15, 115-117.

Javitt, D. C., Steinschneider, M., Schroeder, C. E., Vaughan, H. G., JR., \& ArEzzo, J. C. (1994). Detection of stimulus deviance within primate primary auditory cortex: Intracortical mechanisms of mismatch negativity (MMN) generation. Brain Research, 667, 192-200

Kane, N. M., Curry, S. H., Rowlands, C. A., Manara, A. R., Lewis, T., Moss, T., Cummins, B. H., \& BuTler, S. R. (1996). Eventrelated potentials: Neurophysiological tools for predicting emergence and early outcome from traumatic coma. Intensive Care Medicine, 22, 39-46.

Kaukoranta, E., Sams, M., Hari, R., HämÄlä̈Inen, M., \& NäÄTäNEN, R. (1989). Reactions of human auditory cortex to changes in tone duration: Indirect evidence for duration specific neurons. Hearing Research, 41, 15-22.

KLATT, D. (1980). Software for cascade/parallel formant synthesizer. Journal of the Acoustical Society of America, 67, 971-995.

Kotze, H. F., \& MøLLER, A. T. (1990). Effect of auditory subliminal stimulation on GSR. Psychological Reports, 67, 931-934.
Kraus, N., Koch, D. B., McGee, T. J., Nicol, T. G., \& CunningHAM, J. (1999). Speech-sound discrimination in school-age children: Psychophysical and neurophysiologic measures. Journal of Speech, Language, \& Hearing Research, 42, 1042-1060.

Kraus, N., McGee, T. J., Carrell, T. D., King, C., Littman, T., \& Nicol, T. G. (1994). Discrimination of speech-like contrasts in the auditory thalamus and cortex. Journal of the Acoustical Society of America, 96, 2758-2768.

Kraus, N., McGee, T. J., Carrell, T. D., \& Sharma, A. (1995). Neurophysiologic bases of speech discrimination. Ear \& Hearing, 16, 19-37.

Kraus, N., McGee, T. J., Carrell, T. D., Zecker, S. G., Nicol, T. G., \& KосH, D. B. (1996). Auditory neurophysiologic responses and discrimination deficits in children with learning problems. Science, $\mathbf{2 7 3}$, 971-973.

Kraus, N., McGee, T. J., Littman, T., Nicol, T. G., \& King, C. (1994). Nonprimary auditory thalamic representation of acoustic change. Journal of Neurophysiology, 72, 1270-1277.

Kraus, N., McGee, T. J., Micco, A., Sharma, A., Carrell, T. D., \& NICOL, T. G. (1993). Mismatch negativity in school-age children to speech stimuli that are just perceptibly different. Electroencephalography \& Clinical Neurophysiology, 88, 123-130.

Kraus, N., McGee, T. J., Sharma, A., Carrell, T. D., \& Nicol, T. G. (1992). Mismatch negativity event-related potential elicited by speech stimuli. Ear \& Hearing, 13, 158-164.

Lang, W., Lang, M., Heise, B., Deecke, L., \& Kornhuber, H. H. (1984). Brain potentials related to voluntary hand tracking, motivation and attention. Human Neurobiology, 3, 235-240.

Logan, J., \& PruitT, J. (1995). Methodological issues in training listeners to perceive non-native phonemes. In W. Strange (Ed.), Speech perception and linguistic experience: Issues in cross-language speech research (pp. 351-378). Timonium, MD: York Press.

Macmillan, N. A., \& Creelman, C. D. (1991). Detection theory: A user's guide. Cambridge: Cambridge University Press.

McGee, T. J., Kraus, N., \& Nicol, T. G. (1997). Is it really a mismatch negativity? An assessment of methods for determining response validity in individual subjects. Electroencephalography \& Clinical Neurophysiology, 104, 359-368.

N $\ddot{A} \ddot{A} T \ddot{A} N E N, R .(1990)$. The role of attention in auditory information processing as revealed by event-related brain potentials and other brain measures of cognitive function. Behavioral \& Brain Sciences, 13, 20I-233.

NÄÄTÄNEN, R. (1991). Mismatch negativity outside a strong attentional focus: A commentary on Woldorff et al. Psychophysiology, 28, 478-484.

NäÄTÄNEN, R., Gaillard, A. W., \& Mantysalo, S. (1978). Early selective attention effect on evoked potential reinterpreted. Acta Psychologica, 42, 313-329.

Näätänen, R., Paavilainen, P., Alho, K., Reinikainen, K., \& SAMS, M. (1989). Do event-related potentials reveal the mechanism of the auditory sensory memory in the human brain? Neuroscience Letters, 98, 217-221.

NäÄtänEN, R., \& Picton, T. W. (1987). The N1 wave of the human electric and magnetic response to sound: A review and an analysis of the component structure. Psychophysiology, 24, 375-425.

Novak, G., Ritter, W., Vaughan, H. G., Jr., \& Wiznitzer, M. L. (1990). Differentiation of negative event-related potentials in an auditory discrimination task. Electroencephalography \& Clinical Neurophysiology, 73, 255-275.

Pekkonen, E., Rinne, T., \& NäÄtÄnen, R. (1995). Variability and replicability of the mismatch negativity. Electroencephalography \& Clinical Neurophysiology, 96, 546-554.

PoLICH, J. (1988). Bifurcated P300 peaks: P3a and P3b revisited? Journal of Clinical Neurophysiology, 5, 287-294.

Sams, M., Kaukoranta, E., HämäläInen, M., \& NäÄtänen, R. (1991). Cortical activity elicited by changes in auditory stimuli: Different sources for the magnetic $\mathrm{N} 100 \mathrm{~m}$ and mismatch responses. Psychophysiology, 28, 21-29.

Sams, M., Paavilainen, P., Alho, K., \& NäÄtänen, R. (1985). Auditory frequency discrimination and event-related potentials. Electroencephalography \& Clinical Neurophysiclogy, 62, 437-448.

Sharma, A., Kraus, N., McGee, T. J., Carrell, T. D., \& Nicol, T. G. (1993). Acoustic versus phonetic representation of speech as reflected 
by the mismatch negativity event-related potential. Electroencephalography \& Clinical Neurophysiology, 88, 64-7].

Skrandies, W., \& Jedynak, A. (1999). Learning to see 3-D: Psychophysics and brain electrical activity. NeuroReport, 10, 249-253.

Squires, N. K., Squires, K. C., \& Hillyard, S. A. (1975). Two varieties of long-latency positive waves evoked by unpredictable auditory stimuli in man. Electroencephalography \& Clinical Neurophysiol$o g y, 38,387-401$.

StRANGE, W., \& DitTManN, S. (1984). Effects of discrimination training on the perception of $/ \mathrm{r} /-/ 1 /$ by Japanese adults learning English. Perception \& Psychophysics, 36, 131-145.

TAllal, P., \& STARK, R. E. (1981). Speech acoustic-cue discrimination abilities of normally developing and language-impaired children. Journal of the Acoustical Society of America, 69, 568-574.

TAYlor, M. M., \& CreElman, C. D. (1967). PEST: Efficient estimates on probability functions. Journal of the Acoustical Society of America, 41, 782-787.

Tervaniemi, M., Lehtokoski, A., Sinkkonen, J., Virtanen, J., ILMONIEMI, R. J., \& NÄÄTÄNEN, R. (1999) Test-retest reliability of mis- match negativity for duration, frequency and intensity changes. Clinical Neurophysiology, 110, 1388-1393.

Titinen, H., May, P., Reinikainen, K., \& NäÄtänen, R. (1994). Attentive novelty detection in humans is governed by pre-attentive sensory memory. Nature, 372, 90-92.

Tremblay, K., Kraus, N., Carrell, T. D., \& McGee, T. (1997). Central auditory system plasticity: Generalization to novel stimuli following listening training. Journal of the Acoustical Society of America, 102, 3762-3773.

Tremblay, K., Kraus, N., \& MCGee, T. (1998). The time course of auditory perceptual learning: Neurophysiologic changes during speechsound training. NeuroReport, 9, 3557-3560.

WeRKER, J. F., \& TeES, R. C. (1987). Speech perception in severely disabled and average reading children. Canadian Journal of Psychology, 41, 48-6l.

(Manuscript received July 20, 1999; revision accepted for publication January 17,2000 .) 